\title{
Getting it right the first time: Stress-only MPI in the ER
}

\author{
David G. Wolinsky, MD, FACC, MASNC ${ }^{\mathrm{a}}$ \\ a Heart and Vascular Institute, Cleveland Clinic Florida, Weston
}

Received Feb 7, 2017; accepted Feb 7, 2017

doi: $10.1007 / \mathrm{s} 12350-017-0825-z$

\section{See related article, pp. 1274-1282}

Determining the optimal pathway for evaluating patients presenting to the emergency with chest pain remains an ongoing challenge. The 2011 National Hospital Ambulatory Medical Care Survey reported over seven million emergency room (ER) visits for evaluation of chest pain. ${ }^{1}$ Only a very small percentage of these patients actually have acute coronary syndrome (ACS) ${ }^{2}$ or severe coronary artery disease. Presenting symptoms, electrocardiogram changes, and initial biomarkers can usually identify the small group of patients with acute myocardial infarction (MI) or acute coronary syndromes (ACS). These patients are rapidly admitted and undergo coronary angiography if indicated.

The larger portion of remaining patients is left to undergo diagnostic and prognostic evaluation. These evaluations are usually done in an observation unit or a dedicated chest pain unit with the goal to identify all patients who are at increased short-term risk (48 to 72 hours) of a cardiac event and refer them promptly for care. Additionally, these patients may be at risk for further downstream testing, recurrent hospitalization, or late cardiovascular event. The workup of these patients should minimize these long-term risks. In the present issue of the Journal of Nuclear Cardiology, Amirian et al analyze the utility of both exercise treadmill testing (ETT) and stress-only myocardial perfusion imaging (MPI) in assessing short- and long-term events in a lowrisk population. ${ }^{3}$ Their analysis sheds new light on better options to manage these patients under the pressure of

Reprint requests: David G. Wolinsky, MD, FACC, MASNC, Heart and Vascular Institute, Cleveland Clinic Florida, Weston, USA dwolinsk@gmail.com.

J Nucl Cardiol 2018;25:1283-5.

$1071-3581 / \$ 34.00$

Copyright (C) 2017 American Society of Nuclear Cardiology. new health care delivery models. Incorporating stressonly imaging would assign specific decision-making responsibility to the groups involved in the care of these patients - ER physicians, hospitalists, and cardiologists. By eliminating resting studies in the majority of patients, the evaluation can be completed more quickly and radiation exposure is reduced.

Historically, patients inappropriately discharged from the emergency room with ACS have risk-adjusted mortalities twice that of similar patients who are hospitalized and treated. ${ }^{4}$ In fact, the overall risk in this population is low, but few patients presenting to ER with chest pain are discharged because of medical-legal concerns. As a consequence, these patients undergo exhaustive workups within a 24-48 hour span while hospitalized. From 1999-2000 to 2007-2008, utilization of advanced imaging for evaluation of chest pain increased by $376 \% .^{2}$ Multiple clinical pathways have been developed incorporating biomarkers, functional testing, and noninvasive angiography, all of which have high success rates. ${ }^{5}$ Although patients are no longer admitted, direct costs remain high (\$2226-\$3168) regardless of modality, driven to a great degree by length of stay in the hospital. ${ }^{3}$ In order to reduce the cost, the focus should be on three critical factors-reduce the number of people requiring chest pain pathways, reduce utilization of advanced imaging, or decrease length of hospital stay.

There is a preponderance of data demonstrating that patients with chest pain who do not have a myocardial infarction are at low risk during short- and longer-term follow-up. Foy et al demonstrated in a national sample of over 650,000 patients that the incidence of MI was very low-only $0.11 \%$ at 7 days and $0.33 \%$ at 190 days of follow-up. The incidence of myocardial infarction was not impacted by decision to pursue noninvasive testing prior to discharge nor was the choice of testing a factor. ${ }^{6}$ This analysis was based on insurance claims data; therefore, there was a paucity of clinical data available such as pre-test likelihood of disease. 
Conclusions regarding the true value of testing such as MPI are therefore limited.

Other authors have similar data demonstrating low risk in this population. The Cleveland Clinic has demonstrated that patients who "rule out" for an MI have a low thirty-day event rate. In patients with TIMI scores of 2 or less, stress MPI before discharge adds little prognostic value. In patients with higher TIMI scores, MPI was more likely to be ischemic and coronary angiography was more likely to be carried out; however, revascularization did not significantly improve prognosis. Patients with normal MPI had low cardiac event rates consistent with multiple studies demonstrating excellent prognosis associated with a normal MPI. A Singapore study incorporating SPECT MPI in the ER showed that patients with normal or probably normal (with return for resting images) stress-only MPI were associated with a low 30-day event rate. Incorporating MPI reduced the incidence of coronary angiography significantly and patients with positive MPI were more likely to have significant CAD. ${ }^{\text {? }}$

Present ER evaluation schemes are efficacious at eventually identifying high-risk chest pain patients. However, excessive numbers of patients undergo far too many tests and spend far too many hours in the hospital for these methods to be considered effective. In many centers, initial patient evaluation involves calculation of the TIMI risk score. The TIMI score was designed, however, to assess the risk of patients presenting with acute coronary syndrome/non-ST elevation MI. ${ }^{8}$ Recent studies suggest that applying the HEART score had an improved specificity and sensitivity for predicting MACE when compared to TIMI scores. ${ }^{9}$ HEART scores of less than or equal to three outperformed TIMI scores as a low-risk threshold. ${ }^{10}$ Those patients with the lowest HEART scores could be managed by ER staff. Exercise stress testing, coronary CTA, or other testing could be ordered as indicated.

Hospitalists can manage patents requiring further testing. Exercise stress testing is an appropriate initial test for patients presenting with chest pain. Bourque et al have demonstrated that low-to-intermediate risk patient achieving at least 10 METS with no ischemic ST segment depression have an annual mortality of $0.1 \%$ and myocardial infarction rate of $0.4 \% .{ }^{11}$ Unfortunately, not all patients presenting to emergency room can exercise. Additionally, data from the Duke Treadmill Score database suggest that $64 \%$ of patients do not fall into the low-risk category necessitating further testing. ${ }^{12}$ Consistent with prior studies, Amirian reports a large percentage of low-risk patients of low-risk patients

Logistically, stress-only myocardial perfusion imaging is an ideal test to perform on intermediate to high-risk patients. Patients can be studied with exercise or pharmacologic stress. Resting heart rate does not significantly affect the ability to do a high-quality test. Protocols can be modified for dose reduction or reduced imaging time. For acquisitions that are suboptimal, they can be repeated quickly and easily. Because a resting study is not being carried out, it is reasonable to get two sets of cardiac enzymes and perform stress MPI within 12-18 hours even when patients present to the ER during evening hours. The present study did not specifically compare length of stay using stress-only imaging to alternative imaging tests but did describe historical comparisons suggesting but average length of stay in the present study is much lower than historical findings referenced by Amirian. ${ }^{3}$

It has been reported by multiple authors that normal stress-only MPI carries the same favorable prognosis as a normal rest-stress study for patients being evaluated in the emergency room. ${ }^{13}$ Although these studies were initially carried out on conventional cameras using attenuation correction, a similar favorable prognosis of normal stress-only scan has been reported using prone imaging ${ }^{14}$ or solid-state CZT cameras. ${ }^{15}$

Cardiology consults would only need to be obtained if a stress-only scan is abnormal. Options at that point would be to proceed to coronary angiography, keep patients hospitalized for a later rest image to elucidate the significance of the stress findings, or discharge the patient to return in 24-48 hours for rest images. Completing studies as an outpatient has been shown to be safe. ${ }^{16}$ Amirian confirms that the 72-hour MACE rate $(1.47 \%)$ and death rate $(0 \%)$ were extremely low. ${ }^{3} \mathrm{Lim}$ reports no clinical events over a 30-day period in 131 patients who were discharged to complete their scans as outpatients. ${ }^{16}$ Clinical assessment by cardiology prior to discharge could further reduce the risk of MACE prior to completion of evaluation. Having defined roles for the three physician subgroups should make care more efficient and decrease length of stay. This should reduce the time to ordering MPI studies. Finally, clinical decisionmaking would be expedited resulting in overall decreased cost of hospital stay.

Beyond the initial hospital expenditures, costs can be incurred by repeat hospitalizations and downstream testing, which do not result in improved outcomes. Amirian demonstrated that in his small population, the MPI group had less repeat testing (0.59\%) than the ETT group $(3.53 \%)$. The PROMISE study failed to demonstrate an outcomes benefit of coronary CTA in symptomatic patients with similar chest pain and suspected CAD. ${ }^{17}$ CTA was associated with a higher frequency of coronary angiography and revascularization and higher costs but no improvement in survival. ${ }^{18}$

Recently, the FDA gave preliminary approval to the use of a high-sensitivity troponin assay in emergency 
rooms in the Untied States. While a normal value effectively excludes myocardial infarction, it remains to be seen what effect a positive value will have on ER evaluation. While high values strongly correlate with myocardial infarction and increased risk of events, the fate of those patients with more modest elevations (15$100 \mathrm{ng} / \mathrm{L}$ ) is not clear and further testing is often required. Preliminary data suggest that the use of hs-Tn assay did not decrease resource utilization when compared to standard Tn. ${ }^{19}$ Although time spent in the ER and the use of CTA in the lowest risk patients may be reduced, optimal management of intermediate risk patients will still require efficient imaging protocols.

Cardiovascular disease remains the leading cause of cardiovascular death in the United States. Concern regarding possible myocardial infarction continues to increase the number of patients seeking ER attention for chest pain. Despite a low overall likelihood of CAD, almost all patients undergo some sort of workup including functional or anatomic testing. The use of stress-only myocardial perfusion imaging can effectively direct a smaller number of clinically more appropriate patients to cardiologists for accurate risk stratification and long-term management. Meanwhile, patients with non-coronary chest pain will leave the ER quickly and be referred to relevant consultants efficiently by ER and hospitalist staff.

\section{References}

1. National Center for Health Statistics, National Hospital Ambulatory Medical Care Survey: 2011 Emergency Department Summary Tables. Available at https://www.cdc.gov/nchs/data/ahcd/nhamcs emergency/2011_ed_web_tables.pdf

2. Bhuiya F, Pitts S, McCaig LF. Emergency Department visits for ChestPains and abdominal pain: United states, 1999-2008. NCHS Data Brief. 2010;(43):1-8.

3. Amirian J, Javdan O, Misher J, et al. Comparative efficiency of exercise stress testing with and without stress-only myocardial perfusion imaging in patients with low risk chest pain. J Nucl Cardiol. 2017. doi:10.1007/s12350-016-0774-y.

4. Pope JH, Aufderheide TP, Ruthazer R, et al. Missed diagnosis of acute cardiac ischemia in the emergency department. NEJM. 2000;342:1163-70.
5. Delaney MC, Neth M, Thomas JJ. Chest pain triage: Current trends in the emergency departments in the United States. J Nucl Cardiol. 2016. doi:10.1007/s12350-016-0578-0.

6. Foy AJ, Guodong L, Davidson WR, et al. Comparative effectiveness of diagnostic testing strategies in emergency department patents with chest Pain. An analysis of downstream testing, intervention, and outcomes. JAMA Intern Med. 2015;175:428-36.

7. Cremer PC, Khalef S, Agarwal S, et al. Myocardial perfusion imaging in emergency department patients with negative cardiac biomarkers. Circ Cardiovasc Imaging. 2014;7:912-9.

8. Antman EM, Cohen M, Berninkc PJ, et al. The TIMI risk score for unstable angina /non-ST elevation MI: A method for prognostication and therapeutic decision making. JAMA. 2000;284:835-42.

9. Sakamoto JT, Jiu N, Koh Z, et al. Comparing HEART, TIMI, and GRACE scores for prediction of 30-day major adverse cardiac events in high acuity chest pain patients in the emergency departments. Int Jl Cardiol. 2016;221:759-64.

10. Sun B, Laurie A, Fu R, et al. Comparison of the HEART and TIMI risk scores for suspected acute coronary syndrome in the emergency department. Crit Pathw Cardiol. 2016;15:1-5.

11. Bourque JM, Charlton GT, Holland BH, et al. Prognosis in patients achieving >/10 METS on exercise stress testing: Was SPECT imaging useful? Jl Nucl Cardiol. 2011;230:230-7.

12. Shaw LJ, Peterson ED, Shaw LK, et al. Use of a prognostic treadmill score in identifying diagnostic coronary disease subgroups. Circulation. 1998;98:1622-30.

13. Duvall WL, Wijetunga JR, Klein TM, et al. Stress-only Tc-99m myocardial perfusion imaging in an emergency department chest pain unit. J Emerg Med;42:642-50.

14. Gutstein A, Bental T, Solodky A, et al. Prognosis of stress-only SPECT myocardial perfusion imaging with prone imaging. Jl Nucl cardiol. 2016. doi:10.1007/s12350-016-0617-x.

15. Yokota S, Mouden M, Ottervanger J. Prognostic value of normal stress-only myocardial perfusion imaging: A comparison between conventional and CZT-based SPECT. Eur J Nucl Med Mol Imaging. 2016;43:296-301.

16. Lim SW, Anantharan V, Sundram F, et al. Stress myocardial perfusion imaging for the evaluation and triage of chest pain in re emergency department. J Nucl Cardiol. 2013;20:1003-12.

17. Douglas PS, Hoffman U, Patel MR, et al. Outcomes of anatomical versus functional testing for coronary artery disease. $\mathrm{N}$ Engl $\mathrm{J}$ Med. 2015;372:1291-300.

18. Mark Daniel B, Federspeil Jerome J, Cowper Patricia A. Economic outcomes with anatomical versus functional diagnostic testing for coronary artery disease. Ann Intern Med. 2016. doi: 10.7326/M15-2639.

19. Chew DP, Zeitz C, Worthley M, et al. Randomized comparison of high-sensitivity troponin reporting in undifferentiated chest pain assessment. Circ Cardiovasc Qual Outcomes. 2016;9:1-12. 